\title{
REVIEW
}

\section{MALE BREAST CANCER - CURRENT DIAGNOSTIC APPROACHES}

\author{
A. C. Tutovanu ${ }^{1}$, G. Drăghici², S. M. Oprescu ${ }^{1,3}$ \\ ${ }^{1}$ The University of Medicine and Pharmacy "Carol Davila", Bucharest, Romania \\ ${ }^{2}$ Clinical Nephrology Hospital ’Dr. Carol Davila", Bucharest, Romania \\ ${ }^{3}$ University Emergency Hospital, Bucharest, Romania
}

Corresponding author: Andrei Costin Tutovan

Email: andrei.tutovan@drd.umfcd.ro

\begin{abstract}
Male breast cancer (MBC) is a rare disease even though the incidence has increased in the last two decades. Although breast cancer most affects women, it may occur in males, being diagnosed at later stages. Men with BRCA 2 mutation have a higher risk of developing breast cancer than men in the general population. Diagnostic approaches recommendations are derived from established guidelines for breast cancer in women $(F B C)$. A palpable mass is the most common symptom and the skeletal bones are the most common sites of metastatic recurrence in breast cancer. Male breast cancer remains an uncommon disease. Most studies on men with breast cancer are very rare. The current review was made with an aim to analyze the current diagnostic approaches of male breast cancer patients. Searches were carried out on three electronic databases (Pubmed, Medscape, ScienceDirect) using the title term "Diagnosis of male breast cancer”.
\end{abstract}

KEYWORDS: breast cancer, male, risk factors, diagnosis

\section{INTRODUCTION}

Breast cancer in men $(\mathrm{MBC})$ is a rare malignant disease, only about $1 \%$ of all cases being diagnosed worldwide. Current studies show an increased reporting of it, but still not as high as female breast cancer (FBC), much more frequently diagnosed. The main risk factors, known in the development of $\mathrm{MBC}$ cancer, include: family history, disrupting factors of hormonal androgenic/estrogen balance, genetic mutations (especially those of type BRCA2), age, exposure to radiation for a long period of time, Klinefelter's syndrome [1].

Between 30 and $50 \%$ of male patients known to have breast cancer at an advanced WHO classification of breast tumor stage, will suffer at least one bone metastasis with a significant decrease in quality of life. Only $20 \%$ of patients who have already been diagnosed with at least one bone metastasis will survive more than 5 years. Breast cancer cells once reached at the level of the bone microenvironment, will activate the osteoclasts or/and osteoblasts that will lead to bone destruction or bone apposition [2].

\section{MATERIALS AND METHODS}

This paper aims to make a review of the notions in the literature about the current diagnosis of breast cancer in men and is based on the analysis of the results from the databases: Web of Science, Scopus and Pubmed. The search used the formula: "male breast cancer" AND "diagnosis" and have been filtered according to the language (English) and publication year. 
Mutations of BRCA genes may increase

\section{RESULTS}

\section{Incidence}

The first medical cases of $\mathrm{MBC}$ reported in the literature were mentioned in 1843, describing the cause of death of five Parisian men. According to the estimates of the International Agency for Cancer Research, a division of the World Health Organization, the overall incidence of $\mathrm{MBC}$ reaches to approximately 8,000 cases. At European level, there are approximately 2,750 patients respectively to Americans, where there are about 3750 patients. These data account for about $1 \%$ of the incidence of breast cancer occurring in female sex (FBC), which in turn represents $11,6 \%$ of the total incidence of neoplastic diseases in 2014. The average of the occurrence of breast cancer is approximately 5 years later in the lifespan of male sex versus female [3].

At European level, the annual prevalence is 1 in 100,000 men; these cases representing less than $1 \%$ of the total of patients with malignant disease, this percentage may also be attributed to other comorbidities associated with the patient, such as liver disease and endemic infectious diseases that may affect the level of estrogen in the body. It is reported that approximately $38 \%$ of male patients with breast cancer are also affected by gynecomastia. A positive family history can increase more than 2 times the risk of developing breast cancer. Patients older than 50 years diagnosed with breast cancer have an increased risk of occurrence of mammary neoplasia on the contralateral breast [4].

\section{Risk factors}

The main risk factors involved in the occurrence of MBC are represented by the older age of the patient at the time of diagnosis of mammary neoplasia, family history (relatives who have suffered from breast cancer), Klinefelter syndrome, prolonged exposure to $\mathrm{x}$ and electromagnetic radiation, high levels of estrogen, hyperthyroidism, tuberculosis, mutations in the germinal liners of the androgen receptor (chromosome Xq 11.2 -12) [4]. the risk of developing breast cancer in men. The most common genetic link on the occurrence of $\mathrm{MBC}$ is the mutation occurring in the BRCA2 gene, while the mutation of the BRCA1 gene has a much more limited role in the development of the neoplasm [5].

Gynecomastia is a benign condition that consists of the enlargement of the glandular tissue in men's breast usually bilaterally. The cause of this affection is the hormonal imbalance between estrogen and testosterone. Gynecomastia occurs in approximately $40-65 \%$ of men. Moreover, some recent studies suggest that, when the gynecomastia is installed, the risk of developing MBC is higher [6].

Obesity has been mentioned in several studies, as associated with the occurrence of MBC. Adipose tissue contains the enzyme aromatase, which contributes to the conversion of testosterone into estrogen. The estradiol produced by the aromatization of testosterone, in the adipose tissue suppresses the release of luteinizing hormone, thus producing a decrease in the production of testosterone [7].

Mumps can also affect the level of androgenic hormones (testosterone) and it can lead to the development of male breast cancer over time, but rarely.

Another factor that can trigger $\mathrm{MBC}$ is the regular consumption of alcohol, more than $60 \mathrm{ml}$ per day [8].

\section{Symptoms, manifestations and clinical signs}

The most common symptoms occurring in the MBC consist in the appearance of a firmly painless lump, attached to adjacent tissues in the subareolar region or a non-painful breast tumor mass, of thick tissue with irregular edges, just below the nipple. The presence of gynecomastia can mask the neoplasm. In approximately $75 \%$ of cases, similar to female breast cancer, there is a retraction of the nipple inside and a cutaneous ulceration. The most commonly involved is the left breast, but bilaterality is also reported for $1 \%$ of patients [9].

The mean tumor diameter is between 3 and $3,5 \mathrm{~cm}$, but can range from $0,5 \mathrm{~cm}$ to 12,5 $\mathrm{cm}$. Ulceration or even skin destructions, the attachment of the tumor to the thorax represent the predictive signs of neoplasia, being more 
common in male sex rather than female sex. It may also occur, as in the case of breast cancer in female sex, a rash around the nipple or an unusual redness on certain areas of the skin of the breast, or crust (an orange peel texture).The involvement of axillary lymph nodes, with the presence of adenopathies have been reported in $50 \%$ of patients diagnosed. These facts can be based on the lack of awareness of the male sex on breast neoplasia, men are not instructed to regularly check or palp their breast gland, in order to detect possible signs or lumps, compared to female sex [10].

According to the last specialized studies, men are approximately 1,6 times more likely to have an axillary involvement (lymphadenopathy), compared to women. Remote dissemination may occur with the occurrence of bone metastases, causing both pain and fractures. In the advanced stages, the patient feels uncomfortable, weak and can lose a lot of weight in a short time. The average duration of symptoms, before diagnosis of breast cancer, in male sex was reported between 14 and 21 months in older studies, respectively between one month and 8 months in the latest [11].

Bone metastases resulting from male breast cancer may be associated with a wide range of symptoms, with an increase in morbidity, especially due to severe pain, with the appearance of pathological fractures (sudden occurrence of pain accompanied by the inability of the patient to move) and bone marrow compression (lightning-fast pain accompanied by weakness at the cervical or lumbar level with irradiation in the upper or lower limbs) [12].

The medullary compression of lombosacrate spine may cause horse tail syndrome, a pattern of neuromuscular and urogenital symptoms. This syndrome is characterized by the appearance of lumbar pain or sciatica (irradiated on one pelvic limb, but mostly irradiates on both pelvic limbs), sensory disorders in the sacral region, loss of bladder and intestinal content dysfunction of motor sensitivity and sensitivity in the pelvic limb. Horse tail syndrome is a surgical emergency, because the destruction of nerve roots can be irreversible [13].

Following bone pathological fractures, the level of calcium in the blood reaches a high level, so the patient will accuse the appearance of fatigue, muscle weakness, generalized sick state with nausea, vomiting and dehydration [14].

\section{Diagnosis}

In most cases of male breast cancer, the diagnosis is performed by a triple evaluation: anamnesis, clinical examination by inspection and palpation, respectively by paraclinical methods represented by ultrasound / mammography / MRI / CT / tumor biopsy.

The incidence of breast cancer in male sex is rather low, the use of mammograms as a screening method is not recommended. As a standard, a mammography is performed with two exposures per mammary gland, the oblique medio-lateral exposure, being the most important, respectively, the cranio-caudal exposure. Both of these two exposures are recommended in men who accuse clinical manifestations. Sometimes, for a better assessment of the morphology of detected lesions, it is recommended to perform additional exposures (tangential, enlarged, and centered) [15].

Mammography is an effective diagnostic method with a sensitivity of $92 \%$, respectively with a specificity of $90 \%$. Microcalcifications have irregular spiculated edges, dense irregularities, being uncommon in males. The value of mammography diagnosis can also be influenced by the size, respectively the volume of the mammary gland, the appearance of gynecomastia (appears as a discrete mass), which can be distinguished from the mammary neoplasia [16].

If the result of mammography is suspect and non-conclusive, it is recommended to perform an ultrasonography in order to investigate breast lesions. For deep breast portions and areas that are inaccessible to mammography, it is recommended to use ultrasonography with high frequency transducers. Biopsy guided through mammography cannot be achieved due to the small size of the male breast [17].

Ultrasonography is a useful diagnosis method, because it provides information on nodular involvement, or it can detect invasive cancers, the ones being of a solid nature. Any suspected tumor mass should be biopsied, to 
confirm the diagnosis. The biopsy puncture performed under ultrasound control is preferable, because it can allow a safe, accurate and definitive diagnosis [18].

When a suspicious lesion is discovered both by ultrasonography and by mammography, additional evaluations will be required, consisting of laboratory examinations, pulmonary radiography, bone scintigraphy (for the investigation of bone metastases) and computer tomography (CT). Positron emission tomography (PET-CT) is a more accurate and concise method of diagnostics than $\mathrm{CT}$ for the assessment and identification of small bone metastases, respectively of the lymphatic disseminations, as well as for observing mammary tumor response to chemotherapy [19].

MRI is an important diagnostic method for breast cancer in female sex, but it is rarely used for the males and also referred to as a diagnostic method in the specialty literature. However, it can be successfully used to diagnose neoplastic lesions associated with gynecomastia or invasion of the thoracic wall. Although it can be difficult to achieve technically, a magnetic resonance imaging (MRI) can be successfully used in evaluating and diagnosing equivocal radiological imaging [20].

For bone metastases, the following paraclinical investigations are necessary for diagnosis:

- Bone scintigraphy is very sensitive, but has a low specificity. The 99tc scintigraphy has a sensitivity of approximately $89 \%$, with a false positive rate of about $40 \%$. It is much more sensitive and specific than radiological and computer tomography investigations, but MRI investigation is far superior in the evaluation of vertebral metastases. On scintigraphy, bone metastases appear as multiple outbreaks, hyper-uptake, disseminated to the entire bone skeleton [21].

- Radiography is a cheap, fast, easily accessible technique for evaluating bone metastases. It is the first test to diagnose a sudden bone pain. Radiography is specific, but has a low sensitivity of approximately $44 \%$, since the initial metastatic lesions up to a $1 \mathrm{~cm}$ in size, with a damage of less than $50 \%$ of the trabecular bone may not be radiologically highlighted. Three radiological aspects may be described: the occurrence of osteoblastic metastases, in which the lesion has the form of a bone condense, osteolytic type, in which the lesion causes bone destruction or mixed-type containing both osteoblastic and osteolytics lesions [22].

- Computed Tomography allows the diagnosis of metastatic lesions, with a sensitivity of $71-100 \%$. Bone damage, sclerotic deposits and metastases extension to soft tissues are easy to visualize on CT [23].

- Magnetic resonance is useful in the diagnosis of vertebral metastases, which can be distinguished from infectious processes or spinal osteoporosis. The sensitivity varies between 82 and $100 \%$, and the specificity is between 73 and $100 \%$ [24].

- Positron Emission Tomography (PET) diagnoses the presence of bone metastasis by quantified metabolic activity. It is a superior investigation of bone scintigraphy with a sensitivity of $95 \%$, respectively specificity of $94 \%$ [25].

- Her 2 Test is a test to measure the amount of Her 2 from the neoplastic tissue. The Her2/neu Test is recommended together with those for estrogen and progesterone receptors, as an initial set of analyses, for invasive breast cancer, but can also be performed in the case of recurrent breast cancer. Patients with a positive result in these tests have a reserved prognosis, but these patients will be able to receive the specific treatment of Her2/neu positive cancers. In order to determine whether the breast tumor is positive for Her2 / neu, bone biopsy should be performed with the intention of taking an amount of tumor tissue. There are several tests that can determine the Her2 status:

1. Immunohistochemical test (IHC) 
2. In situ fluorescent hybridization Test (FISH)

3. In situ chromogenic hybridization Test (CISH)

4. Enhanced silver-situ hybridization Test (SISH)

Her2/neu rapid tests using blood as a sample of determination are also available on the market [26].

These tests are not conclusive and are not used for screening purposes, but may help to determine the prognosis and to monitorize the treatment. If the immunohistochemical test (IHC) is positive, the Her2/neu gene overexpresses the Her2 protein. If the FISH test is positive, Her2/neu gene is amplified. If one of these two tests is positive, the patient has an aggressive breast tumor with a poor response to the endocrine treatment, resistant to chemotherapy, but with response to trastuzumab treatment. If the FISH test is positive, but the IHC test is negative, the breast tumor still responds to trastuzumab treatment. If both tests are negative, treatment with trastuzumab will not have any effect [27].

\section{Differential diagnosis}

Differential diagnosis of male breast cancer, can be done with a wide variety of diseases, including benign affections (gynecomastia), with metastatic malignant processes in the mammary gland (melanoma, lymphoma, lung cancer), as well as various pathologies of inflammatory nature [28].

Lymphoma is a disease with a primary or secondary malignant nature that develops at the level of the lymphatic system. The most common are secondary types, non-Hodgkin Bcell lymphomas. Its manifestation is represented by the appearance of palpable and painless tumor masses. In the mammogram examination, the appearance of several solitary or multiple tumor masses is well-defined and the lymph nodes are present, in both the mammary and axillary levels. At the ultrasound, there are observed a single or multiple solid tumor mass, respectively multiple abnormal lymph nodes with cortical thickening. Most of the time, primary lymphoma can imitate the imaging of a primary malignancy of the mammary gland [29].
The most common and frequent (up to $65 \%$ ) condition of the breast occurring in the male sex is gynecomastia, which is described as a subareolar mobile mass [30].

Imagistically, subareolar glandular tissue can be visualized and sometimes considered asymmetric. Ecographically, the glandular tissue found at the nipple level has a predominantly hyperecogenic structure. Gynecomastia can have both an avascular and a hypervascular structure at the Doppler color exam, depending on the stage of its development [31].

Another differential diagnosis is pseudodogynecomastia, a disorder without usually palpable mass, which results from the fat related associated with obesity. Imagistically this is well circumscribed both ultrasound and mammographically [32].

Mastitis with abscesses formation is another differential diagnosis, which is manifested by the occurrence of fever, erythema, sensitivity and thickening of the skin. Mammographically, mastitis presents an increase in the unilateral mammary gland with the appearance of a thickening of the skin [33].

Abscesses are represented on mammography, as irregular forms with or without calcifications, very difficult to differentiate from a neoplastic process [34].

Necrosis of fat tissues resulting from a strong trauma, with or without the occurrence of calcifications, constitutes a good differential diagnosis of breast cancer, because this disease also manifests itself by the appearance of destructive or skin necrosis. On mammography can be diagnosed, as centrally occurring calcifications or as the appearance of wellcircumscribed radiotransparent formations, while on ultrasound these can occur as both hypoechogenic and hyperechogenic masses [35].

Breast cysts are formations occurring in the mammary gland, with their own round wall, containing liquid and they develops from the ducto-lobular structures of the mammary gland. Cysts can be presented both as groups consisting of small cysts and as solitary subcutaneous formations, organized in placards, unilateral or bilateral. They may also be included in the differential diagnosis of breast cancer, such as other subcutaneous lesions, 
hematoma and lipoma [36]. On mammography, breast cysts are typically presented as superficial masses well delimited presenting low density. Ultrasound can have a homogeneous or heterogeneous structure, often presenting the echographic sign in the "claw" [37].

On mammography, the hematoma appears well circumscribed or irregular and it can be quite difficult to differentiate them from a neoplastic process. On ultrasound, they are hyperechogenic and variable in appearance over time, because they can become hypoechogenic [38].

On mammography, lipoma appears radiotransparently, being surrounded by a radiopaque capsule and on ultrasound they are well circumscribed with a homogeneous structure [39].

\section{CONCLUSIONS}

The overall awareness of male sex, as regards the risk of developing breast cancer, is minimal, which leads to a delay in the diagnosis, so the prognosis is much more reserved than in the case of female sex. Breast cancer in men is a problem, which should be tackled more seriously, both by the medical and public community. According to the literature, more than $40 \%$ of men diagnosed with breast cancer are in a terminal stage, with a minimum life expectancy. Male sex is diagnosed with breast cancer at an older age than female sex, the risk of neoplastic cell dissemination and mortality will increase concurrently, with the development of breast cancer. Therefore, for an early diagnosis of breast cancer, the same screening methods used in the case of female sex are required in the male sex.

\section{REFERENCES}

[1]RM Ferzoco, Kh. J. Ruddy, "Optimal Delivery Of Male Breast Cancer Follow-Up Care: Improving Outcomes Breast Cancer," Dove Med Pres. Published Online, pp. 371-379, Nov 2015.

[2]CS Shemanko, Yi. Cong, F.Al. Desiderio, "What Is Breast In The Bone?," Academic Editorint J Mol Sci. Published Online, pp. 1764, Oct 2016.

[3]MP Humphries, V.Cr. Jordan,Va.Speirds, "Obesity And Male Breast Cancer: Provocative Parallels," Bmc Med. Published Online, pp.113-134, Jun 2015
[4]M Yalaza, Ay Inan, M. Bozer, "Male Breast Cancer,"J Breast Health. Published Online, pp. 1-8, Jan.2016.

[5]VM Basham, JM Lipscombe, JM Ward, et al. "BRCA1 and BRCA2 mutations in a populationbased study of male breast cancer," Breast Cancer Res, R2. pp.4, Jan 2002.

[6]R Gennari, g. Gurigliano, BA. Jereczek-Fossa, et al "Male breast cancer: a special therapeutic problem. Anything new? ,“ Int J Cancer. pp. 663-70, Feb 2004.

[7]Z Nahleh, S Girnius, "Male breast cancer: a gender issue," Nat

Clin Pract Oncol. pp. 428-437, Aug 2006.

[8].Ka Kanchan, G.S. Rodrigues, M. Kulkarni, "Male Breast Cancer: Is The Scenario Changing?," World J Surg Oncol. Published Online. pp 52-58, Jun 2008.

[9]L Fiala, O Coufal, V Fait, et al. "Male breast cancer: our experience," Rozhl. Chir. pp. 612-618, Sept. 2010.

[10]SH Giordano, Ds Cohen, Au Buzdar, et al, "Breast carcinoma in men: a population-based study," Cancer. pp 51-57, Feb 2004.

[11]Re Coleman. "Metastatic bone disease: clinical features, pathophysiology and treatment strategies," Cancer Treatment Reviews. pp. 165-176, Oct 2001.

[12]Mj Goblirdch, Pp Zwolak, Dr Clohisy "Biology of bone cancer pain,"Clinical Cancer Research. Pt.2. pp 6231s-6235s, Apr 2006.

[13]M Cecchini, A Wetterwald, G Pluijm, et al, "Molecular and biological mechanisms of bone metastasis," EAU Update Series. pp.214-226, Feb 2005.

[14]R Maisano, S Pergolizzi, S Cascinu "Novel therapeutic approaches to cancer patients with bone metastasis," Crit Rev Oncol Hematol, pp.239-250 Jan 2001.

[15]Ma Jafari, A Olfatbakhsh, Ki Rezaei-Kalantari "A Review Of Risk Factors And Clinical And Radiological Features," Male Breast Cancer Volume 1, Issue 4 pp.25-30, Octt 2017.

[16]Lg Menezes, M Knuttel, L Stehouwer, et al "Magnetic Resonance Imaging In Breast Cancer: A Literature Review And Future Perspectives," World J Clin Oncol, Published Online. Pp.61-70, May 2014.

[17]K Kelly, J Dean, Ws Comulada, Sj Lee "Breast cancer detection using automated whole breast ultrasound and mammography in radiographically dense breasts," Eur Radiol. pp.734-742, Aug 2010.

[18]C Balleyguier, P Opolon, MC Mathieu, et al "New potential and applications of contrastenhanced ultrasound of the breast: Own investigations and review of the literature," European journal of radiology. pp.14-23, Dec 2009. 
[19]P Lind, I Igerc, T Beyer, et al "Advantages and limitations of FDG PET in the follow-up of breast cancer,"European journal of nuclear medicine and molecular imaging. pp.125-134, Oct 2004.

[20]N Houssami, RM Turner, M Morrow "Metaanalysis of pre-operative magnetic resonance imaging (MRI) and surgical treatment for breast cancer," Breast Cancer Res Treat. pp. 273-283, Nov 2017.

[21]V Cuccurullo, GL Cascini, O Tamburrini "Bone metastases radiopharmaceuticals: an overview," Curr Radiopharm, pp. 41-47, Feb 2013.

[22]J Chop, M. Raghavan "Diagnostic imaging and image-guided therapy of skeletal metastases," Cancer Control.pp. 102-112. Jun 2012.

[23]V Vassiliou, D Andreopoulos, S Frangos, et al "Bone metastases: assessment of therapeutic response through radiological and nuclear medicine imaging modalities," Clin Oncol (R Coll Radiol),pp. 632-645, Nov 2011.

[24]HL Yang, XM Wang, SM Deng "Diagnosis of bone metastases: a meta-analysis comparing "18FDG PET, CT, MRI and bone scintigraphy,“,Eur Radiol. pp. 2604-2617, Mar 2011.

[25]Yu HH, YY Tsai, SE Hoffe. "Overview of diagnosis and management of metastatic disease to bone," Cancer Control. pp 84-91, Dec 2012.

[26]C Gutierrez, R Schiff "Her 2: Biology, Detection, And Clinical Implications," Arch Pathol Lab Med pp. 55-62, Jan 2011.

[27]P OA, R Guzman, M Cervantes, A Santiago. "Characterization of HER2 status by fluorescence in situ hybridization (FISH) and immunohistochemistry (IHC)," Methods Mol Biol. 1180(chapter 10), Press MF, pp. 181-207, Feb 2014. [28]I Fentiman "Male breast cancer: a review," Ecancermedicalscience. pp.140, Mar 2009.

[29]G.E Jr Lattin, R A Jesinger, L M Glassman "From the radiologic pathology archives: diseases of the male breast: radiologic-pathologic correlation," Radiographics. pp. 461-489, Feb 2013.

[30]KY Şafak "Mammography Findings of Male Breast Diseases," The Journal of Breast Health. pp.106-110, April 2015.

[31]V Dialani, J Baum, T S Mehta "Sonographic features of gynecomastia," J Ultrasound Med. pp.539-547, Jun 2010.

[32]MB Popli, V Popli, P Bahl, Y Solanki. "Pictorial essay: Mammography of the male breast," The Indian Journal of Radiology \& Imaging. pp.278-281, Jan 2009.

[33]F Draghi, CC Tarantino, L Madonia, G Ferrozzi. "Ultrasonogra- phy of the male breast," J Ultrasound. pp.122-129, Feb 2011.

[34]T Kazama, I Tabei, C Sekine. "Subareolar breast abcess in male patients: a report of two patients with a literature review," Surgical case reports. pp.121128, Dec2017.

[35]E Iuanow, M Kettler, PJ Slanetz.. " Spectrum of disease in the male breast," AJR Am J Roentgenol. pp.247-259, May 2011.

[36]S Yitta, CI Singer, HB Toth, CL Mercado "Image presentation. Sonographic appearances of benign and malignant male breast disease with mammographic and pathologic correlation," J Ultrasound Med. pp. 931-947, Oct 2010.

[37]M. Rosa, S Masood. "Cytomorphology of male breast lesions: diagnostic pitfalls and clinical implications," Diagn Cytopathol. pp.179-184, Feb 2012.

[38]C Nguyen,M D Kettler, M E Swirsky, et al “ Male breast disease: pictorial review with radiologic-pathologic correlation," Radiographics. pp.763-779, May 2013.

[39]L Chen, P K Chantra, et al "Imaging characteristics of malignant lesions of the male breast,",Radiographics. pp.993-1006, Feb. 2006. 